Revista Arbitrada Interdisciplinaria KOINONIA

Año 2020. Vol V. N5. Especial II: Educación

Hecho el depósito de Ley: FA2016000010

ISSN: 2542-3088

FUNDACIÓN KOINONIA (F.K). Santa Ana de Coro. Venezuela.

Rodrigo Giovani Aguirre-Herráez; Darwin Gabriel García-Herrera; Claudio Fernando Guevara-Vizcaíno; Juan Carlos Erazo-Álvarez

http://dx.doi.org/10.35381/r.k.v5i5.1052

\title{
Realidad aumentada y educación en el Ecuador
}

\section{Augmented reality and education in Ecuador}

Rodrigo Giovani Aguirre-Herráez

rodrigo.aguirre.81@est.ucacue.edu.ec

Universidad Católica de Cuenca, Gualaquiza

Ecuador

https://orcid.org/0000-0001-6213-4600

Darwin Gabriel García-Herrera

dggarciah@ucacue.edu.ec

Universidad Católica de Cuenca, Azogues

Ecuador

https://orcid.org/0000-0001-6813-8100

Claudio Fernando Guevara-Vizcaíno

cquevarav@ucacue.edu.ec

Universidad Católica de Cuenca, Cuenca

Ecuador

https://orcid.org/0000-0003-3593-0606

Juan Carlos Erazo-Álvarez

jcerazo@ucacue.edu.ec

Universidad Católica de Cuenca, Cuenca

Ecuador

https://orcid.org/0000-0001-6480-2270

Recibido: 15 de septiembre de 2020

Revisado: 19 de octubre de 2020

Aprobado: 15 de noviembre de 2020

Publicado: 01 de diciembre de 2020 
Rodrigo Giovani Aguirre-Herráez; Darwin Gabriel García-Herrera; Claudio Fernando Guevara-Vizcaíno; Juan Carlos Erazo-Álvarez

\title{
RESUMEN
}

La investigación tuvo por objetivo analizar la realidad aumentada y educación en el Ecuador desde una revisión crítica de literatura científica. De tipo descriptiva apoyada en un diseño bibliográfico. El $57.1 \%$ de encuestados no utilizan la Realidad Aumentada por falta de conocimiento, el $32.1 \%$ por falta de equipos tecnológicos para ponerlo en práctica dentro de las instituciones educativas, $10.7 \%$ de los encuestados indica que no utilizan esta herramienta por causa del acceso limitado a internet. Hay que impulsar la preparación continua de los docentes en las nuevas tecnologías pues tienen que tener la capacidad para impartir estos conocimientos en los alumnos, buscando su mejor desenvolvimiento en el ámbito tecnológico, el mismo evoluciona tan rápido y se convierte en un elemento importante dentro de las actividades humanas, la educación y la tecnología son elementos que tiene que desarrollarse paralelamente pues los dos se constituyen pilares importantes de la sociedad.

Descriptores: Inteligencia artificial; Aplicación informática; Tecnología educacional. (Palabras tomadas del Tesauro UNESCO).

\begin{abstract}
The objective of the research was to analyze augmented reality and education in Ecuador from a critical review of scientific literature. Descriptive type supported by a bibliographic design. $57.1 \%$ of respondents do not use Augmented Reality due to lack of knowledge, $32.1 \%$ due to lack of technological equipment to put it into practice within educational institutions, $10.7 \%$ of respondents indicate that they do not use this tool due to limited access to Internet. It is necessary to promote the continuous preparation of teachers in new technologies because they have to have the ability to impart this knowledge to students, seeking their best development in the technological field, it evolves so quickly and becomes an important element within human activities, education and technology are elements that have to develop in parallel, since both are important pillars of society.
\end{abstract}

Descriptors: Artificial intelligence; computer applications; educational technology. (Words taken from the UNESCO Thesaurus). 


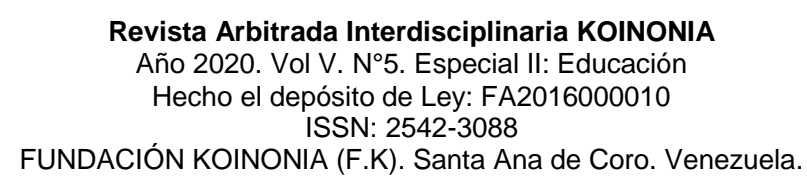

Rodrigo Giovani Aguirre-Herráez; Darwin Gabriel García-Herrera; Claudio Fernando Guevara-Vizcaíno; Juan Carlos Erazo-Álvarez

\section{INTRODUCCIÓN}

La realidad aumentada es una tecnología relativamente nueva, pero con gran acogida en la actualidad, nos presenta varias bondades en diferentes campos, en la educación permite incorporar mayor cantidad de elementos a la información que se quiere transmitir a los estudiantes, ya que añade información de tipo virtual en un plano real, generando nuevos puntos de vista y una información más completa, que mejora la comprensión de lo que se desea transmitir. (Fombona-Cadavieco \& Pascual-Sevillano, 2017).

La tecnología es un aliado importante de la realidad aumentada pues van juntas en el desarrollo y su aplicación, al incorporar nuevos descubrimientos, mejoras en los aparatos tecnológicos y nuevos software, enfocados en el uso de la realidad aumentada se revelan nuevas utilidades de la misma, en la educación es fácil reproducir elementos de tres dimensiones por medio de los móviles que son usados como herramienta para mejorar el proceso enseñanza-aprendizaje. (Blázquez-Sevilla, 2017).

La realidad aumentada es considerada de gran importancia pues lo fácil de su aplicación y el interés que ha despertado en los investigadores por los beneficios y lo moldeable que es, da como resultado nuevos descubrimientos y aplicaciones para utilizarla en los diferentes campos de la vida humana, en la educación nos presenta más de 200 aplicaciones en los diferentes sistemas operativos móviles, que son de fácil uso y en la mayoría de los casos gratuitos. (Marín-Díaz \& Sampedro-Requena, 2020).

Los dispositivos móviles son parte importante de nuestra vida están presentes en todas las actividades que realizamos, se considera la puerta de entrada para mejorar el proceso de enseñanza-aprendizaje porque permite a los usuarios mediante sus aplicaciones interactuar con varias fuentes de información, que enriquece el conocimiento del usuario, esto sumado a la realidad aumentada es una poderosa herramienta para incrementar el interés de los estudiantes. (Organista-Sandoval, et al., 2018).

La realidad aumentada es superponer elementos virtuales en un plano real, lo que permite al usuario tener una información más completa del objeto de estudio, sin 


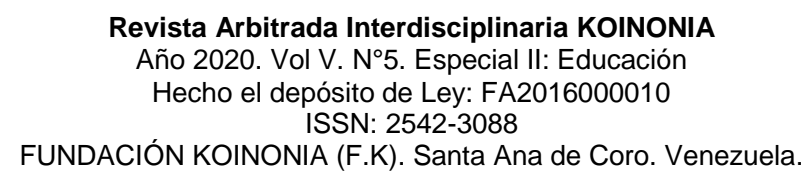

Rodrigo Giovani Aguirre-Herráez; Darwin Gabriel García-Herrera; Claudio Fernando Guevara-Vizcaíno; Juan Carlos Erazo-Álvarez

embargo, los proyectos creados como realidad aumentada no han dejado de crecer en los últimos años, todos aportan con criterios distintos dependiendo el campo de aplicación, la realidad aumentada no remplaza al mundo real más bien lo enriquece.

Según varios autores el uso de la realidad aumentada en la educación incrementa el interés y la curiosidad de los estudiantes por el uso de nuevas tecnologías capaces de romper la forma histórica de impartir conocimientos, es aquí donde existe un gran reto para los docentes que debemos dejar de lado la forma tradicional y recurrir a nuevos modelos de educación que tienen como principal aliado la tecnología. (Angarita-López, 2018).

La tecnología y la educación van de la mano, es por ello que usar realidad aumentada en las aulas está en auge, la facilidad de contar con dispositivos capaces de reflejar elementos virtuales en un entorno real, han creado la necesidad de evaluar las ventajas de la realidad aumentada como instrumento de enseñanza, estos estudios dan resultados similares evaluándolo de una manera cuantitativa y cualitativa, queda demostrado que usar esta tecnología genera mayor interés por parte del alumnado. (Méndez-Muros, 2018).

La realidad aumentada está aplicada en la educación, turismo, marketing, videojuegos, etc. Es una tecnología que presenta varias utilidades, en el Ecuador se han realizado varias investigaciones y creado aplicaciones dentro de las universidades que ayudan a mejorar la comprensión de su alcance, tener la posibilidad de dejar de lado la educación tradicional y buscar mejores oportunidades de enseñanza es lo que hace la diferencia. (Henne \& Schmidt-Kraepelin, 2015).

En razón de lo planteado, la investigación tuvo por objetivo analizar la realidad aumentada y educación en el Ecuador desde una revisión crítica de literatura científica. 


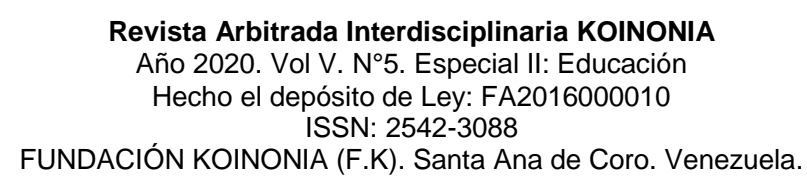

Rodrigo Giovani Aguirre-Herráez; Darwin Gabriel García-Herrera; Claudio Fernando Guevara-Vizcaíno; Juan Carlos Erazo-Álvarez

\section{Referencial teórico}

La realidad aumentada nace en la década de 1960 pero el primer sistema formal se desarrolló en los años 90. Desde entonces se han realizado diferentes estudios, tesis, conferencias y simposios de rango internacional sobre la realidad aumentada y sus posibles beneficios, los investigadores se sienten atraídos por la gran ventaja que presenta esta tecnología y las utilidades de la misma dentro de varios campos.

¿Qué es la Realidad Aumentada?

El concepto más utilizado sobre Realidad Aumentada es dada por Milgram y Kishino en el año 1994 quienes indican que: "entre un entorno real y un entorno virtual puro esta la llamada realidad mixta y esta se subdivide en dos, la realidad aumentada (más cercana a la realidad) y la virtualidad aumentada más próxima a la virtualidad pura)" (MeloBohórquez, 2018). La realidad aumentada es aquella que tiene elementos virtuales, pero está basada en lo real, mientras la realidad virtual es aquella creado por tecnología informática dando al usuario la percepción de ser parte de este entorno. A partir de esta definición, se han elaborado cambios, adaptaciones y utilidades dentro de varios campos en los cuales esta herramienta es de gran ayuda.

(Asaad, et al., 2019), indican que la realidad aumentada está basada en un mundo real pero ampliada por un computador lo que genera imágenes del mundo real pero en un plano virtual, además para considerarse realidad aumentada deberían existir elementos en 3D. La realidad aumentada es un instrumento interactivo que posee como base la información del mundo real y añade nuevos elementos procedente de lo virtual, esto ayuda a que la información sea más integra. En este proceso los conocimientos que el usuario tiene del mundo real son potenciados, hay que recalcar que esta herramienta está considerada dentro de las 10 tecnologías más importantes a partir del 2008 pues, despierta el interés de los investigadores por las ventajas que presta y sus aplicaciones en muchas áreas del conocimiento. (Cubillo-Arribas, et al., 2014).

Por su parte (Anancolla, 2018). Afirman que "la realidad aumentada no reemplaza el mundo real por uno virtual, sino al contrario, mantiene el mundo real que ve el usuario 


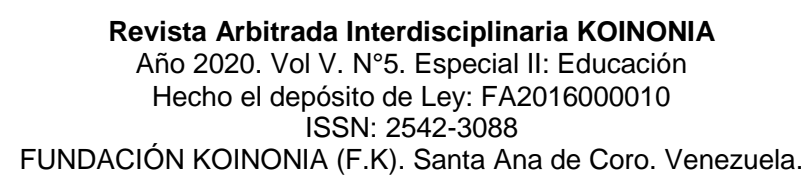

Rodrigo Giovani Aguirre-Herráez; Darwin Gabriel García-Herrera; Claudio Fernando Guevara-Vizcaíno; Juan Carlos Erazo-Álvarez

complementándolo con información virtual superpuesto al real". El beneficiario nunca pierde la relación con el mundo real, pero es capaz de interactuar con objetos virtuales y estudiarlo desde perspectivas que no serían posibles en un plano real.

Las características que tienen que cumplir los elementos para ser considerados realidad aumentada es: componerse de elementos virtuales y reales, interacción en tiempo real e información almacenada en 3D. Para ejecutar la realidad aumentada se necesitan de las siguientes tareas principales, captar e identificar la escena, ser visualizado, combinar lo real y virtual; este último potencia el objeto de estudio (Andres, et al., 2018). En la actualidad la realidad aumentada ofrece un sin número de interpretaciones y está presente en muchas áreas, mostrando nuevas maneras de manejar y transmitir la información existente.

\section{Beneficios de la Realidad Aumentada}

Los resultados obtenidos en las investigaciones señalan que la utilización de la realidad aumentada como estrategia de aprendizaje fomenta la interacción con lo virtual, permiten obtener mejores resultados en la formación de los estudiantes dentro del proceso de aprendizaje, a la vez los alumnos se sienten motivados por el uso de nuevas tecnologías. La utilidad de la realidad aumentada va incrementando dentro de la educación en disciplinas prácticas y es muy importante pues le permite al usuario proyectar objetos y contenidos en tres dimensiones. Esto posibilita a los estudiantes tener una mejor perspectiva de los que se está investigando, además despierta su interés pues todo se realiza de un modo interactivo. Ha alcanzado un protagonismo importante en muchas áreas del conocimiento pues su versatilidad y otras ventajas que presenta son utilizadas en procesos educativos y de training a nivel mundial. (López, 2014).

A partir de esto aparecen nuevas líneas de investigación basada en la realidad aumentada y los beneficios que genera su aplicación en las diferentes actividades humanas, así en el mundo aparecen varios autores que nos indican. Que es significativo el diseño de aulas virtuales pues beneficia a estudiantes que por su estilo de aprendizaje 


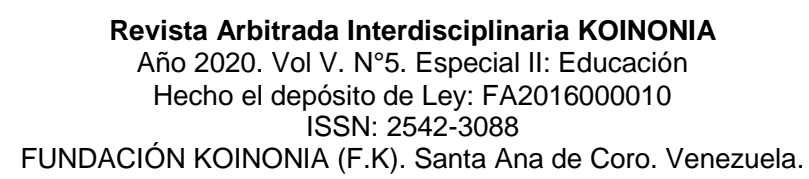

Rodrigo Giovani Aguirre-Herráez; Darwin Gabriel García-Herrera; Claudio Fernando Guevara-Vizcaíno; Juan Carlos Erazo-Álvarez

y rasgos de personalidad presentan bajos rendimientos y dificultades para adaptarse a algunos entornos educativos, esta tecnología provoca una mayor participación de los estudiantes y una mejora en el rendimiento académico. (Quizhpi-Lupercio, 2018).

Con todo esto se puede asegurar que la teoría de la realidad aumentada puede mejorar la relación entre los humanos y el mundo virtual, por cuanto el uso de los ordenadores es importante en la mayoría de las actividades cotidianas, posibilitando el acceso a todo tipo de usuarios desde principiantes hasta expertos, dado que los objetos comunes se convierten en elementos atractivos dentro de lo virtual. Los estudios realizados indican que el sistema de enseñanza a través de la realidad aumentada genera mayor interés, pues proporciona al usuario una información más detallada de los objetos de estudio, potenciando el aprendizaje de habilidades prácticas, comprensión e investigación, además que aumenta la curiosidad por el uso de dispositivos móviles dentro de las aulas como medio de aprendizaje. (Rico-González \& Quevedo, 2016).

(Méndez-Muros, 2018), indican que la realidad aumentada al ser incluida en la enseñanza educativa, se ha podido identificar algunos beneficios como: el aumento del interés de los estudiantes por su autoaprendizaje, potencia el aprendizaje por medio de juegos y genera interacción en tiempo real, permite conectar lo intelectual con la experiencia física, mejorando la asimilación y comprensión.

Implementar la realidad aumentada en la educación ecuatoriana representa un desafío por parte de las instituciones educativas, por que conlleva gastos e inversión en recursos tecnológicos, humanos y materiales, además que la realidad socio económico de algunos alumnos no les permite adquirir el equipo necesario para su aprendizaje. Sin embargo se debe realizar un programa de implementación progresivo de esta herramienta, debido a que sus beneficios son mayores a sus desventajas (Hernández-Revelo, 2018).

Tomando en cuenta estas investigaciones la realidad aumentada podría generar grandes ventajas en las diferentes asignaturas en especial en áreas como la ciencia e ingeniería, pues esta herramienta esta creada para trabajar de una forma práctica dentro del aula, produce motivación extra en los estudiantes porque les lleva de un plano real a uno mixto, 


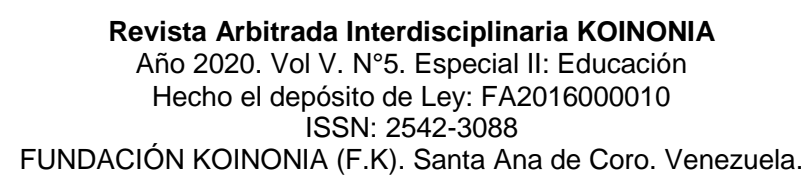

Rodrigo Giovani Aguirre-Herráez; Darwin Gabriel García-Herrera; Claudio Fernando Guevara-Vizcaíno; Juan Carlos Erazo-Álvarez

lo que les permite mejorar su perspectiva sobre los objetos o lugares que se estudian, además que mejoran sus conocimientos referente al uso de la tecnología, aplicaciones y software.

La realidad aumentada permite al usuario incrementar las experiencias del entorno real por medio de un ordenador, lo que facilita la proyección de objetos y lugares de la realidad a un ámbito tecnológico, en dimensiones como la 3D permitiendo su estudio en diferentes perspectivas que no fueran posibles en el plano real. (Andres, et al., 2018).

La realidad aumentada ayuda a eliminar algunas barreras existentes en la educación. Se puede realizar de manera virtual experimentos que por sus costos no son posibles en la realidad mientras que en el espacio virtual siempre están disponibles. Se puede proyectar las reacciones de los experimentos que son peligrosos sin riesgos, rompe el límite del tiempo y es posible observar los cambios de la materia y objetos que en la realidad serían largos en un corto tiempo, asi mismo, se apoya en los elementos del mundo real pero enriquecido con características tecnológicas generado por un software, esta información es más compleja pues se puede analizar desde puntos de vista que antes no eran posibles.

\section{Tecnología móvil y Realidad Aumentada}

Considerando el rápido avance de la tecnología es necesario mantener su ritmo y realizar inversiones en el área educativa que permitan, a los estudiantes y docentes tener un acceso a las nuevas tecnologías. Igual de importante es que estas herramientas sean de calidad para que el proceso enseñanza-aprendizaje se lleve de una mejor manera y los docentes puedan aplicarla en su práctica educativa (Varela, et al., 2018). Otra tecnología que ha cambiado nuestra forma de vida, es la tecnología móvil y es la que debemos apuntar para enfocarla en el ámbito educativo, puesto que cuenta con varias utilidades y aplicaciones que serían de gran ayuda, combinándola con la Realidad Aumentada permite experiencias que no se podían dar y hoy en día están a nuestro alcance. 


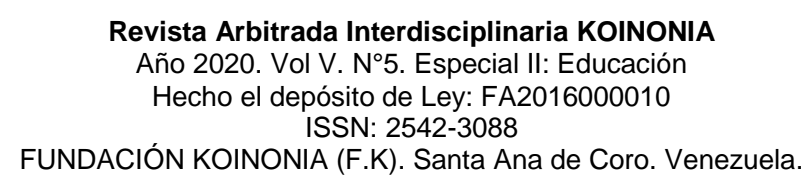

Rodrigo Giovani Aguirre-Herráez; Darwin Gabriel García-Herrera; Claudio Fernando Guevara-Vizcaíno; Juan Carlos Erazo-Álvarez

Los libros y apuntes constituyen un pilar fundamental en la educación pero con el paso del tiempo van perdiendo su efectividad, pues el desarrollo tecnológico crece rápidamente lo que genera nuevas herramientas que tienen mayor acogida por profesores y estudiantes, porque hacen más efectivo el proceso de enseñanza y aprendizaje. La Realidad Aumentada se convierte en modelo de interacción para visualizar un objeto o espacio físico virtual en tiempo real, combinando el mundo real con objetos digitales, permiten fidelizar a los usuarios. Teniendo en cuenta que los sistemas computacionales se encuentran presentes en casi todas las actividades humanas, esto nos da una percepción de que existe una brecha muy pequeña entre lo real y lo virtual. Esta metodología de enseñanza, ha incrementado sus beneficios para los educadores debido a los dispositivos tecnológicos existentes y sus aplicaciones, pues son de fácil manejo, lo que permite mejorar la didáctica fusionando lo teórico y lo práctico, trae consigo desventajas relacionadas al ámbito económico por la falta de recursos para adquirir los equipos necesarios con el fin de aplicar la realidad aumentada, la escasez de conocimientos por parte de los docentes para implementarla dentro del aula.

En la actualidad la facilidad de poseer aparatos tecnológicos y estar conectados todo el tiempo a internet, el sin número de aplicaciones que nos ayuda a desenvolvernos en las tareas diarias, se han hecho parte esencial de nuestra vida; es así que la realidad aumentada va teniendo mayor acogida dentro de la educación pues permite combinar estas dos herramientas tecnológicas para mejorar la enseñanza dentro del aula (PérezLisboa, et al., 2020). La realidad aumentada nace en la década de 1960 pero el primer sistema formal se desarrolló en los años 90. Desde entonces se han realizado diferentes estudios, tesis, conferencias y simposios de rango internacional sobre la realidad aumentada y sus posibles beneficios, los investigadores se sienten atraídos por la gran ventaja que presenta esta tecnología y las utilidades de la misma dentro de varios campos. 


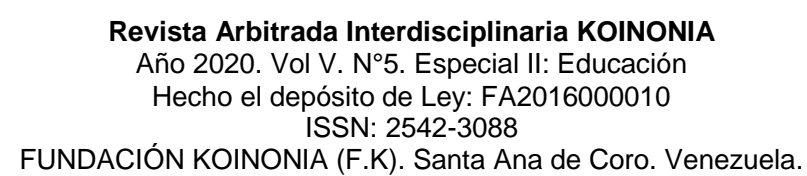

Rodrigo Giovani Aguirre-Herráez; Darwin Gabriel García-Herrera; Claudio Fernando Guevara-Vizcaíno; Juan Carlos Erazo-Álvarez

\section{¿Qué es la Realidad Aumentada?}

El concepto más utilizado sobre Realidad Aumentada es dada por Milgram y Kishino en el año 1994 quienes indican que: "entre un entorno real y un entorno virtual puro esta la llamada realidad mixta y esta se subdivide en dos, la realidad aumentada (más cercana a la realidad) y la virtualidad aumentada más próxima a la virtualidad pura)" (MeloBohórquez, 2018).

La realidad aumentada es aquella que tiene elementos virtuales, pero está basada en lo real, mientras la realidad virtual es aquella creado por tecnología informática dando al usuario la percepción de ser parte de este entorno. A partir de esta definición, se han elaborado cambios, adaptaciones y utilidades dentro de varios campos en los cuales esta herramienta es de gran ayuda. (Asaad, et al., 2019), indica que la realidad aumentada está basada en un mundo real pero ampliada por un computador lo que genera imágenes del mundo real pero en un plano virtual, adeás para considerarse realidad aumentada deberían existir elementos en 3D.

La realidad aumentada es un instrumento interactivo que posee como base la información del mundo real y añade nuevos elementos procedente de lo virtual, esto ayuda a que la información sea más integra. En este proceso los conocimientos que el usuario tiene del mundo real son potenciados, hay que recalcar que esta herramienta está considerada dentro de las 10 tecnologías más importantes a partir del 2008 pues, despierta el interés de los investigadores por las ventajas que presta y sus aplicaciones en muchas áreas del conocimiento. (Cubillo-Arribas, et al. 2014).

Por su parte (Anancolla, 2018). Afirman que "la realidad aumentada no reemplaza el mundo real por uno virtual, sino al contrario, mantiene el mundo real que ve el usuario complementándolo con información virtual superpuesto al real". El beneficiario nunca pierde la relación con el mundo real, pero es capaz de interactuar con objetos virtuales y estudiarlo desde perspectivas que no serían posibles en un plano real.

Las características que tienen que cumplir los elementos para ser considerados realidad aumentada es: componerse de elementos virtuales y reales, interacción en tiempo real e 


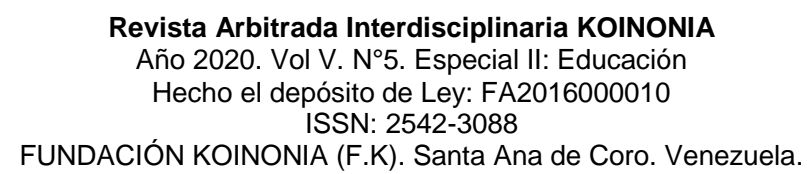

Rodrigo Giovani Aguirre-Herráez; Darwin Gabriel García-Herrera; Claudio Fernando Guevara-Vizcaíno; Juan Carlos Erazo-Álvarez

información almacenada en 3D. Para ejecutar la realidad aumentada se necesitan de las siguientes tareas principales, captar e identificar la escena, ser visualizado, combinar lo real y virtual; este último potencia el objeto de estudio. (Andres, et al., 2018). En la actualidad la realidad aumentada ofrece un sin número de interpretaciones y está presente en muchas áreas, mostrando nuevas maneras de manejar y transmitir la información existente.

\section{Beneficios de la Realidad Aumentada.}

Los resultados obtenidos en las investigaciones señalan que la utilización de la realidad aumentada como estrategia de aprendizaje fomenta la interacción con lo virtual, permiten obtener mejores resultados en la formación de los estudiantes dentro del proceso de aprendizaje, a la vez los alumnos se sienten motivados por el uso de nuevas tecnologías. La utilidad de la realidad aumentada va incrementando dentro de la educación en disciplinas prácticas y es muy importante pues le permite al usuario proyectar objetos y contenidos en tres dimensiones. Esto posibilita a los estudiantes tener una mejor perspectiva de los que se está investigando, además despierta su interés pues todo se realiza de un modo interactivo. Ha alcanzado un protagonismo importante en muchas áreas del conocimiento pues su versatilidad y otras ventajas que presenta son utilizadas en procesos educativos y de training a nivel mundial. (López, 2014).

A partir de esto aparecen nuevas líneas de investigación basada en la realidad aumentada y los beneficios que genera su aplicación en las diferentes actividades humanas, así en el mundo aparecen varios autores que nos indican. Que es significativo el diseño de aulas virtuales pues beneficia a estudiantes que por su estilo de aprendizaje y rasgos de personalidad presentan bajos rendimientos y dificultades para adaptarse a algunos entornos educativos, esta tecnología provoca una mayor participación de los estudiantes y una mejora en el rendimiento académico. (Quizhpi-Lupercio, 2018).

Con todo esto se puede asegurar que la teoría de la realidad aumentada puede mejorar la relación entre los humanos y el mundo virtual, por cuanto el uso de los ordenadores es 


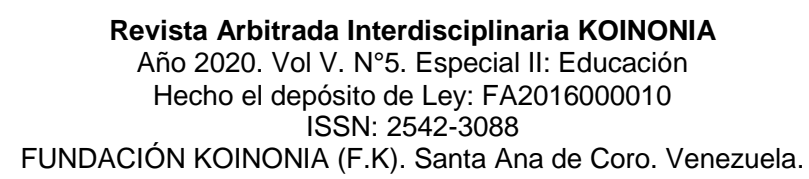

Rodrigo Giovani Aguirre-Herráez; Darwin Gabriel García-Herrera; Claudio Fernando Guevara-Vizcaíno; Juan Carlos Erazo-Álvarez

importante en la mayoría de las actividades cotidianas, posibilitando el acceso a todo tipo de usuarios desde principiantes hasta expertos, dado que los objetos comunes se convierten en elementos atractivos dentro de lo virtual. Los estudios realizados indican que el sistema de enseñanza a través de la realidad aumentada genera mayor interés, pues proporciona al usuario una información más detallada de los objetos de estudio, potenciando el aprendizaje de habilidades prácticas, comprensión e investigación, además que aumenta la curiosidad por el uso de dispositivos móviles dentro de las aulas como medio de aprendizaje (Rico-González \& Quevedo, 2016).

(Méndez-Muros, 2018), indican que la realidad aumentada al ser incluida en la enseñanza educativa, se ha podido identificar algunos beneficios como: el aumento del interés de los estudiantes por su autoaprendizaje, potencia el aprendizaje por medio de juegos y genera interacción en tiempo real, permite conectar lo intelectual con la experiencia física, mejorando la asimilación y comprensión. Implementar la realidad aumentada en la educación ecuatoriana representa un desafío por parte de las instituciones educativas, por que conlleva gastos e inversión en recursos tecnológicos, humanos y materiales, además que la realidad socio económico de algunos alumnos no les permite adquirir el equipo necesario para su aprendizaje. Sin embargo se debe realizar un programa de implementación progresivo de esta herramienta, debido a que sus beneficios son mayores a sus desventajas (Hernández-Revelo, 2018).

Tomando en cuenta estas investigaciones la realidad aumentada podría generar grandes ventajas en las diferentes asignaturas en especial en áreas como la ciencia e ingeniería, pues esta herramienta esta creada para trabajar de una forma práctica dentro del aula, produce motivación extra en los estudiantes porque les lleva de un plano real a uno mixto, lo que les permite mejorar su perspectiva sobre los objetos o lugares que se estudian, además que mejoran sus conocimientos referente al uso de la tecnología, aplicaciones y software.

La realidad aumentada permite al usuario incrementar las experiencias del entorno real por medio de un ordenador, lo que facilita la proyección de objetos y lugares de la realidad 


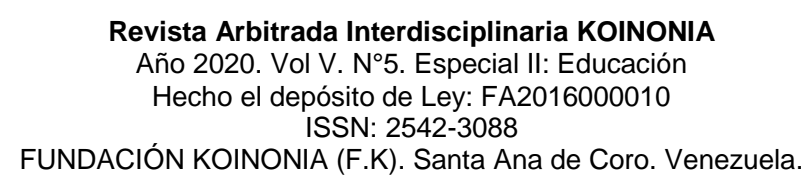

Rodrigo Giovani Aguirre-Herráez; Darwin Gabriel García-Herrera; Claudio Fernando Guevara-Vizcaíno; Juan Carlos Erazo-Álvarez

a un ámbito tecnológico, en dimensiones como la 3D permitiendo su estudio en diferentes perspectivas que no fueran posibles en el plano real. (Andres, et al., 2018). La realidad aumentada ayuda a eliminar algunas barreras existentes en la educación. Se puede realizar de manera virtual experimentos que por sus costos no son posibles en la realidad mientras que en el espacio virtual siempre están disponibles. Se puede proyectar las reacciones de los experimentos que son peligrosos sin riesgos, rompe el límite del tiempo y es posible observar los cambios de la materia y objetos que en la realidad serían largos en un corto tiempo. (Cazar \& González, 2017). La realidad aumentada se apoya en los elementos del mundo real pero enriquecido con características tecnológicas generado por un software, esta información es más compleja pues se puede analizar desde puntos de vista que antes no eran posibles.

\section{Tecnología móvil y Realidad Aumentada.}

Considerando el rápido avance de la tecnología es necesario mantener su ritmo y realizar inversiones en el área educativa que permitan, a los estudiantes y docentes tener un acceso a las nuevas tecnologías. Igual de importante es que estas herramientas sean de calidad para que el proceso enseñanza-aprendizaje se lleve de una mejor manera y los docentes puedan aplicarla en su práctica educativa (Varela, et al., 2018). Otra tecnología que ha cambiado nuestra forma de vida, es la tecnología móvil y es la que debemos apuntar para enfocarla en el ámbito educativo, puesto que cuenta con varias utilidades y aplicaciones que serían de gran ayuda, combinándola con la Realidad Aumentada permite experiencias que no se podían dar y hoy en día están a nuestro alcance.

Los libros y apuntes constituyen un pilar fundamental en la educación pero con el paso del tiempo van perdiendo su efectividad, pues el desarrollo tecnológico crece rápidamente lo que genera nuevas herramientas que tienen mayor acogida por profesores y estudiantes, porque hacen más efectivo el proceso de enseñanza y aprendizaje. La Realidad Aumentada se convierte en modelo de interacción para visualizar un objeto o espacio físico virtual en tiempo real, combinando el mundo real con 


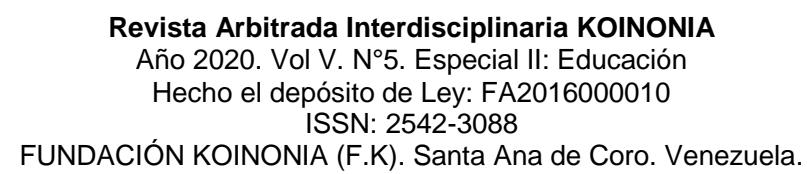

Rodrigo Giovani Aguirre-Herráez; Darwin Gabriel García-Herrera; Claudio Fernando Guevara-Vizcaíno; Juan Carlos Erazo-Álvarez

objetos digitales, permiten fidelizar a los usuarios. Teniendo en cuenta que los sistemas computacionales se encuentran presentes en casi todas las actividades humanas, esto nos da una percepción de que existe una brecha muy pequeña entre lo real y lo virtual. Esta metodología de enseñanza, ha incrementado sus beneficios para los educadores debido a los dispositivos tecnológicos existentes y sus aplicaciones, pues son de fácil manejo, lo que permite mejorar la didáctica fusionando lo teórico y lo práctico, trae consigo desventajas relacionadas al ámbito económico por la falta de recursos para adquirir los equipos necesarios con el fin de aplicar la realidad aumentada, la escasez de conocimientos por parte de los docentes para implementarla dentro del aula.

En la actualidad la facilidad de poseer aparatos tecnológicos y estar conectados todo el tiempo a internet, el sin número de aplicaciones que nos ayuda a desenvolvernos en las tareas diarias, se han hecho parte esencial de nuestra vida; es así que la realidad aumentada va teniendo mayor acogida dentro de la educación pues permite combinar estas dos herramientas tecnológicas para mejorar la enseñanza dentro del aula (PérezLisboa, et al., 2020).

La realidad aumentada es una tecnología que está lista para quedarse en el entorno educativo pues todos los días se le adjudican beneficios y aplicaciones, esto sumado al gran interés que despierta en los estudiantes, crea una herramienta eficiente para impartir conocimiento. Solo por citar, en el sistema Android existen más de 200 aplicaciones de realidad aumentad, muchos autores afirman que la realidad aumentada ayuda a mejorar la práctica Educativa. (Prendes-Espinoza, 2015).

\section{METODOLOGÍA}

Metodológicamente la investigación es de tipo descriptiva no experimental transversal, apoyada en un diseño bibliográfico que permitió la revisión documental de trabajos investigativos relacionados al tema con el propósito de describir las ventajas que presenta la realidad virtual para la educación. 
Rodrigo Giovani Aguirre-Herráez; Darwin Gabriel García-Herrera; Claudio Fernando Guevara-Vizcaíno; Juan Carlos Erazo-Álvarez

Para obtener los datos estadísticos se tomó una muestra de 28 estudiantes de la maestría en Educación, Tecnología e Innovación del segundo corte paralelo B de la Universidad Católica de Cuenca - Ecuador, a quienes se les aplicó una encuesta enfocada en la Realidad Aumentada y su aplicación dentro de las aulas, buscando conocer si esta metodología es aplicada, los beneficios y dificultades de ponerlo en práctica, mediante un instrumento tipo cuestionario de opciones de respuestas en escala Likert, el cual fue validado por juicio de expertos y se calculó el Alfa de Cronbach, con resultados de 0,89 siendo confiable para su aplicación. Los datos obtenidos fueron procesados por la estadística descriptiva, chi cuadrado de Pearson y análisis de contenido documental.

\section{RESULTADOS}

Se describen los resultados obtenidos:

\section{Tabla 1.}

¿Cree que existen beneficios al utilizar Realidad Aumentada en el aula? ¿Haz utilizado Realidad Aumentada como herramienta para tu clase?

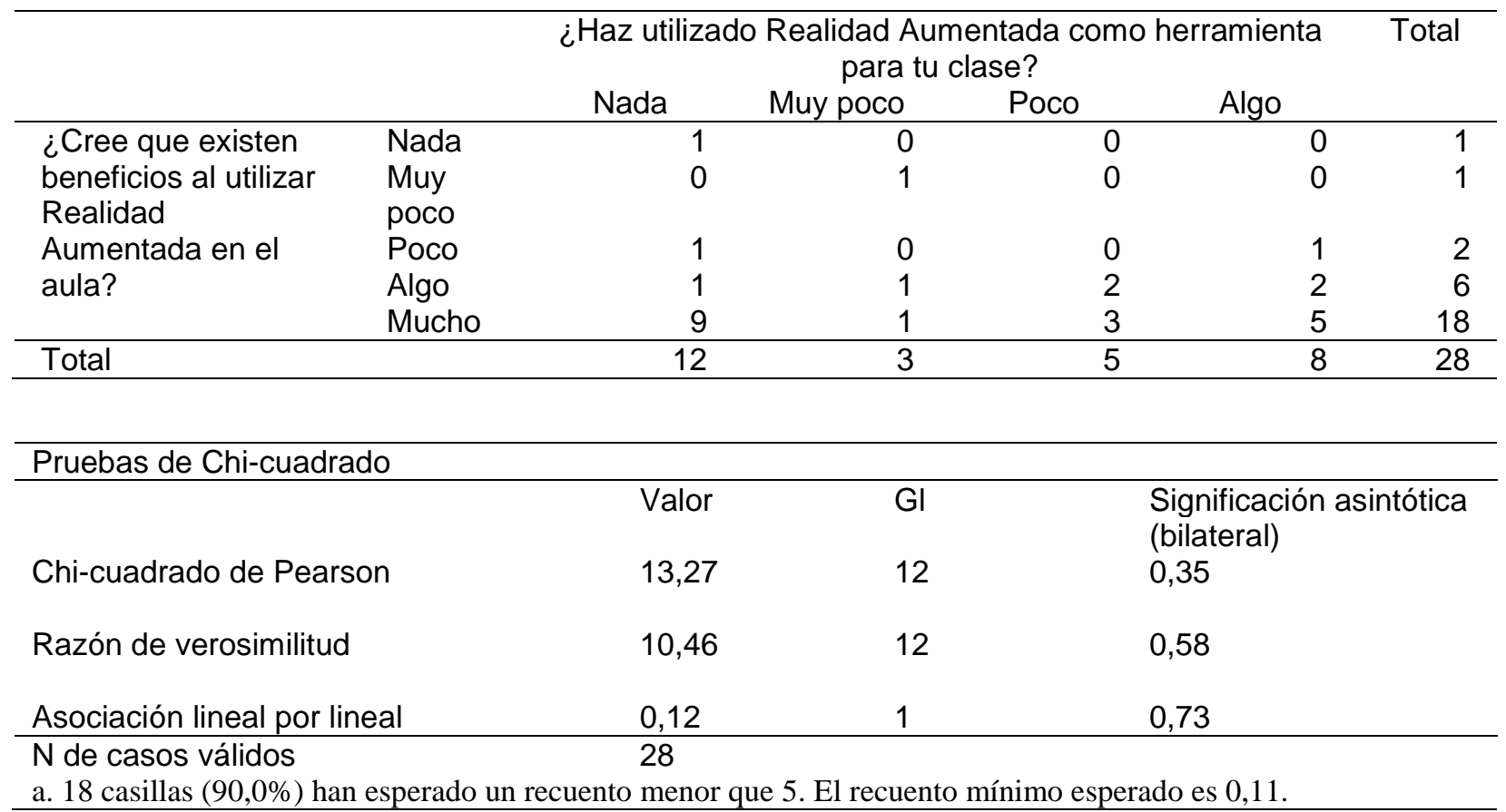

Fuente: Elaboración Propia. 
Rodrigo Giovani Aguirre-Herráez; Darwin Gabriel García-Herrera; Claudio Fernando Guevara-Vizcaíno; Juan Carlos Erazo-Álvarez

El Cuadro estadístico anterior indica que 12 encuestados creen que existe muchos beneficios al utilizar realidad aumentada en sus aulas pero no la han utilizado, 3 persona creen que existe beneficios al utilizar realidad aumentada pero han utilizado muy poco, 5 creen que si existe beneficios de utilizar Realidad Aumentada pero la han utilizado poco y 8 encuestado cree que existe beneficios de utilizar realidad aumentada en sus clases pero han aplicaron solo algo, este cuadro nos demuestra que las variables son inversas que indican que los encuestados conocen de los beneficios de la realidad aumentada pero no se pone en práctica dentro de las aulas, el Chi-cuadrado de Pearson nos indica que las dos variables son independientes, la Realidad Aumentada ayuda a desarrollar el interés de los estudiantes dentro de las instituciones educativas pero no es aplicada por la mayor parte de los encuestados.

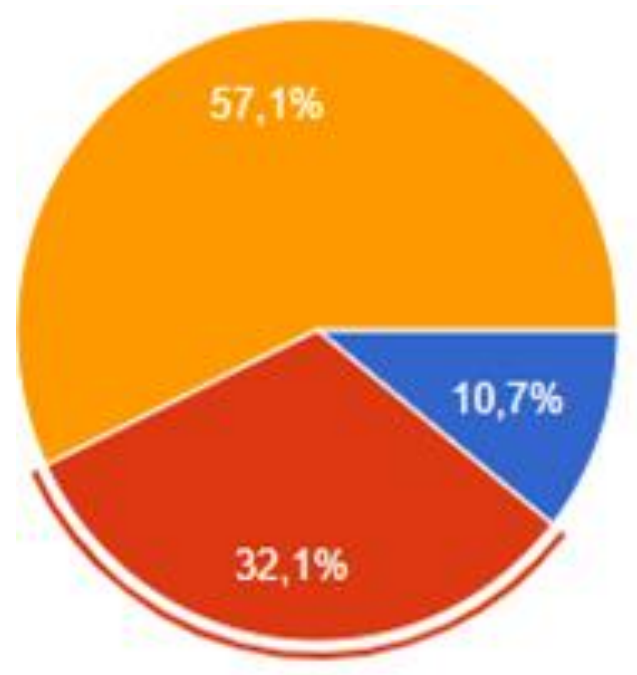

Internet

- Falta de Equipos

Falta de conocimiento de esta herramienta

Figura 1. Desafíos de implementar Realidad Aumentada.

Fuente. Elaboración propia. 


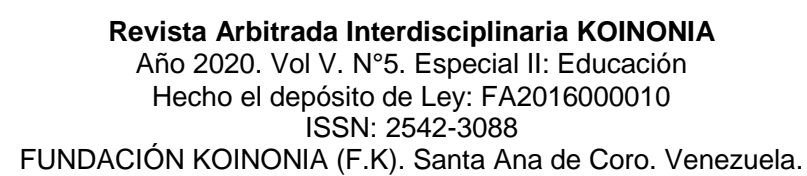

Rodrigo Giovani Aguirre-Herráez; Darwin Gabriel García-Herrera; Claudio Fernando Guevara-Vizcaíno; Juan Carlos Erazo-Álvarez

La siguiente gráfica explica que el $57.1 \%$ de encuestados no utilizan la Realidad Aumentada por falta de conocimiento, el $32.1 \%$ por falta de equipos tecnológicos para ponerlo en práctica dentro de las instituciones educativas, $10.7 \%$ de los encuestados indica que no utilizan esta herramienta por causa del acceso limitado a internet, con estos resultados queda claro que el gran problema es la falta de conocimiento de la Realidad Aumentada como herramienta para el proceso de enseñanza-aprendizaje.

\section{Analisis documental - bibliográfico}

Se realizó una revisión bibliográfica de los elementos que contenían como tema o descriptores la Realidad Aumentada y educación con archivo PDF en idioma español, se encontró cerca de 322000 documentos e investigaciones con elementos de realidad aumentada a nivel global, al realizar la investigación dentro del Ecuador nos arroja cerca de 101000 resultados de investigaciones y documentos en el internet acerca de Realidad Aumentada enfocada en la educación, esta tecnología es realmente nueva pero su gran versatilidad y usabilidad hacen que sea interesante para los investigadores, es por ello que cuenta con gran cantidad de información acerca de su aplicación en los diferentes campos de la humanidad.

(Prendes-Espinoza, 2015). Nos presenta varias experiencias de realidad aumentada, indica que proporciona a los alumnos una mejor comprensión de los contenidos de las asignaturas así como un interés más elevado del estudiante por el manejo de nuevas tecnologías, dentro del aula menciona algunas alternativas de herramientas como realitat3, BuildAR, 3DSMax, Environmental detectives, Eduloc y códigos QR que son software, juegos y elementos que ayudan al docente a implementar la Realidad Aumentada como una herramienta eficiente e interactiva para mejorar el proceso de enseñanza.

(Angarita-López, 2018), da a conocer varios estudios de Realidad Aumentada aplicada a la educación, incluso en niños con Trastorno del Espectro Autista (TEA) realizado en la ciudad de Quito, se realizaron creaciones de libros de Realidad Aumentada, así como 


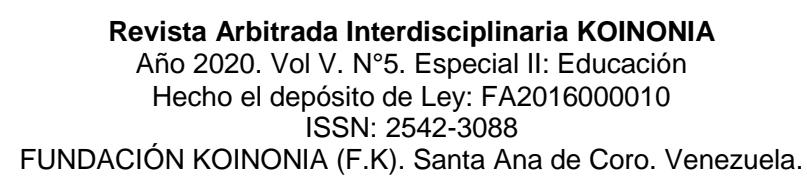

Rodrigo Giovani Aguirre-Herráez; Darwin Gabriel García-Herrera; Claudio Fernando Guevara-Vizcaíno; Juan Carlos Erazo-Álvarez

informes de ejecución y cumplimento de objetivos, al aplicar esta herramienta el resultado obtenido es que, la Realidad Aumentada ayuda a mejorar el entendimiento de los temas de estudio y aumento de motivación del alumnado.

La Realidad Aumentada en Educación Primaria desde la visión de los estudiantes, es un estudio orientado a demostrar las factibilidades del uso de esta tecnología, en ella los estudiantes están totalmente de acuerdo en las ventajas que presenta, destacando el incremento de la creatividad y el uso de los teléfonos inteligentes como herramienta en el desarrollo de sus clases. (Marín-Díaz \& Sampedro-Requena, 2020).

El incremento de accesibilidad a internet trae consigo nativos digitales capaces de manejar la red, programas y aplicaciones con una gran facilidad, tener información de toda índole y explicada desde diferentes perspectivas, se debe complementar con un docente capaz de guiar el uso de esta herramienta y conseguir que la tecnología sea un aliado para mejorar la enseñanza, la Realidad Aumentada como metodología debe ser acompañado de didáctica capaz de llegar al alumno y llamar su atención (Zamora, 2016). (Fombona-Cadavieco \& Pascual-Sevillano, 2017), explican que una educación virtual es una buena opción, sin embargo, es mejor una mezcla entre la tecnología y la clase tradicional, pues permite que exista una guía y un control en el aula, evitando distracción por el manejo de las nuevas tecnologías, hacen énfasis que el manejo de Realidad Aumentada sumado a los dispositivos móviles generan entornos colaborativos que permite una mejor recepción de conocimientos que se pueden impartir a toda hora y en cualquier lugar.

Las aplicaciones, la tecnología móvil y su fácil uso según, Zuckerberg (CEO de Facebok) "Estamos convirtiendo la cámara en la primera plataforma a gran escala de Realidad Aumentada" (18/04/2017). Predecir qué pasará con esta nueva tecnología, a donde nos puede llevar es realmente incierto, en la educación es una herramientas con grandes beneficios, pero a su vez un desafío de acoplar en la clase, hoy en día se habla ya de híper realidad que estará presente en todas las actividades realizadas por el hombre mejorando la información de objetos y teorías (Blázquez-Sevilla, 2017). 


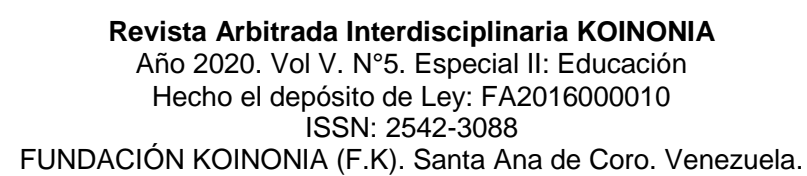

Rodrigo Giovani Aguirre-Herráez; Darwin Gabriel García-Herrera; Claudio Fernando Guevara-Vizcaíno; Juan Carlos Erazo-Álvarez

En la Universidad San francisco de Quito se realizó una investigación de tipo práctica creando estaciones de realidad aumentada para generar experiencias en esta tecnología y mostrar las ventajas que presenta, se indica que es de fácil uso y genera una gran curiosidad en el alumnado al interactuar con objetos digitales rompiendo las limitaciones de tiempo y espacio, lo que mejora la experiencia del aprendizaje. (Varela, et al., 2018). Según la investigación efectuada por (Rico-González \& Quevedo, 2016). Expone que la aplicación de la realidad aumentada potencia la motivación de los estudiantes pero que no se puede dejar de lado el aporte y control de parte del educador, si un alumno está motivado y con alto interés es fácil llegar a un conocimiento significativo al crear una clase interactiva con información generada por un ordenador que suma elementos virtuales a la realidad conlleva a un proceso de aprendizaje eficiente.

\section{PROPUESTA}

Con el paso del tiempo, acceso a internet menos limitado y aparatos moviles con mayor capacidad de reproducir elementos virtuales dejan claro que el avance tecnológico debe ir de la mano con el desarrollo educativo, pues las instituciones educativas son quienes preparan a los futuros profesionales que necesitan conocer de las actualizaciones existentes en el mundo, ser capacez de desemvolverce dentro del ambito laboral con herramientas tecnológicas que estan presentes en todas las actividades realizadas por el_hombre.

Se recomienda el uso de la aplicación Blippar para generar experiencias en el manejo de Realidad Aumentada en estudiantes de bachillerato, por que se necesita de teléfonos inteligentes de mediana gama, esta herramienta realiza el escaneo de documentos en el cual los docentes pueden crear sus contenidos de manera gratuita en plataforma como android e ios, esta herramienta es gratis y los contenidos se pueden compartir por el lapso de 60 días, permite al usuario impartir los contenidos de una clase en una sola imagen en el cual se puede incorporar videos, galerías e información en pdf, con solo 


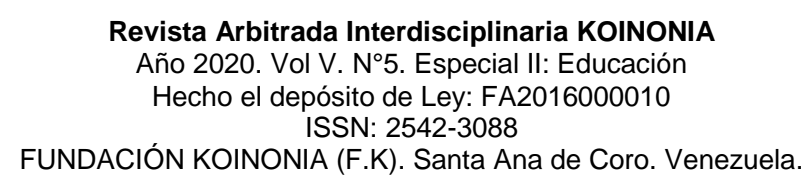

Rodrigo Giovani Aguirre-Herráez; Darwin Gabriel García-Herrera; Claudio Fernando Guevara-Vizcaíno; Juan Carlos Erazo-Álvarez

escanear la imagen que proporciona el docente se accede a los contenidos del tema que desea exponer.

El compromiso del docente del siglo XXI debe ser la preparación para dar respuesta a las incertidumbres presentadas por los alumnos en el manejo de las nuevas tecnologías para que sirven y cual es el verdadero alcance de estas herramientas sus ventajas, aplicaciones y como nos servirá en un futuro profesional, es por ello que debe existir una capacitación continua hacia el docente en manejo de tecnología, hacer una socialización en el ámbito educativo de los beneficios que presenta la Realidad Aumentada, crear la necesidad en el educador de cambiar su forma tradicional de impartir conocimientos, estar actualizado y satisfacer las necesidades de sus alumnos digitales, ser parte del cambio es la unica manera de mejorar la educación.

Se propone el uso de la aplicación Quiver en niños, es una aplicación de Realidad Aumentada gratuita en la cual se permite a los niños colorear en plantillas animadas en 3D, estas plantillas se puede encontrar de forma gratuita en el buscador Google y la aplicación está disponible para todos los dispositivos moviles, al terminar de colorear escanea las plantillas, esta herramienta permite al niño percibir los sonidos y movimientos de los objetos de estudio generando un mayor interes por los contenidos que se desea transmitir.

\section{CONCLUSIONES}

El uso de realidad aumentada mejora el aprendizaje, el estudiante es el protagonista y creador de sus conocimientos, se propone impulsar la utilización de esta tecnología por parte del docente, se requiere incorporar elementos virtuales en las aulas lo que trae consigo elevado interés por el manejo de las nuevas tecnologías, que deben estar presentes dentro del currículo, promoviendo su desarrollo para el desempeño en el ámbito laboral que exige una preparación acorde al avance tecnológico.

Los resultados obtenidos en las encuestas muestran que los docentes conocen los beneficios de la Realidad Aumentada pero la mayoría no lo pone en práctica por falta de 
Rodrigo Giovani Aguirre-Herráez; Darwin Gabriel García-Herrera; Claudio Fernando Guevara-Vizcaíno; Juan Carlos Erazo-Álvarez

conocimiento acerca de esta herramienta, incorporar Realidad Aumentada dentro de las aulas es un reto, pero se garantiza el incremento del interés por parte del alumnado pues la clase se vuelve más práctica y autodidáctica.

Con la revisión bibliográfica del texto se encontró en los artículos y trabajos de investigación una particularidad, en todas ellas se indica que la Realidad Aumentada ayuda como herramienta para mejorar el aprendizaje en diferentes niveles de educación, con lo que se puede asegurar que la realidad aumentada es eficiente para llegar alcanzar aprendizajes significativos, en todas las investigaciones que se exploró se recomienda el uso de aplicaciones o crear contenidos propios de Realidad Aumentada.

Hay que impulsar la preparación continua de los docentes en las nuevas tecnologías pues tienen que tener la capacidad para impartir estos conocimientos en los alumnos, buscando su mejor desenvolvimiento en el ámbito tecnológico, el mismo evoluciona tan rápido y se convierte en un elemento importante dentro de las actividades humanas, la educación y la tecnología son elementos que tiene que desarrollarse paralelamente pues los dos se constituyen pilares importantes de la sociedad.

\section{FINANCIAMIENTO}

No monetario

\section{AGRADECIMIENTO}

A la Universidad Católica de Cuenca; por apoyar el desarrollo de la investigación. 
Revista Arbitrada Interdisciplinaria KOINONIA

Año 2020. Vol V. N5. Especial II: Educación

Hecho el depósito de Ley: FA2016000010

ISSN: 2542-3088

FUNDACIÓN KOINONIA (F.K). Santa Ana de Coro. Venezuela.

Rodrigo Giovani Aguirre-Herráez; Darwin Gabriel García-Herrera; Claudio Fernando Guevara-Vizcaíno; Juan Carlos Erazo-Álvarez

\section{REFERENCIAS CONSULTADAS}

Anancolla, M. L. (2018). Realidad Aumentada como apoyo al proceso enseñanzaaprendizaje en la Unidad Educativa Fray Bartolomé de las Casas-Salasaca. [Augmented Reality as support for the teaching-learning process in the Fray Bartolomé de las Casas-Salasaca Educational Unit]. Ambato: Universidad Regional Autónoma de los Andes. Obtenido de http://dspace.uniandes.edu.ec/handle/123456789/8156

Andres, D., Martinez, C., \& Rosales, R. (2018). Propuesta de aplicación de realidad aumentada utilizando dispositivos inteligentes como herramienta de apoyo a las prácticas del laboratorio de hardware de la Universidad Tecnológica de El Salvador. [Proposed augmented reality application using smart]. San Salvador: Universidad Tecnológica de El Salvador. Obtenido de https://n9.cl/afrq4

Angarita-López, J. (2018). Apropiación de la realidad aumentada como apoyo a la enseñanza de las ciencias naturales en educación basica primaria. [Apropiation of increased reality as a support to the teaching of Natural Sciences in primary basic]. Universidad Pedagógica y Tecnológica (Colombia). Recurso en línea. Tomo 17, páginas https://repositorioslatinoamericanos.uchile.cl/handle/2250/3176058 279-300).

Asaad, R., Sulaiman, Z., \& Abdulmajeed, S. (2019). Proposed System for Education Augmented Reality Self English Learning. Academic Journal Of Nawroz University, 8(3), 27-32. https://doi.org/10.25007/ajnu.v8n3a366

Blázquez-Sevilla, A. (2017). Realidad aumentada en Educación. [Augmented Reality in Education]. Monografía (Manual). Rectorado (UPM). http://oa.upm.es/45985/

Cazar, P. J., \& González, P. M. (2017). Realidad aumentada en el proceso de enseñanzaaprendizaje en la asignatura de Ciencias Naturales, unidad 4 de décimo año de EGB, en la unidad educativa Gran Bretaña, periodo 2016-2017. [Augmented reality in the teaching-learning process in the Natural Sciences subject, unit 4 of the tenth year of EGB, in the Great Britain educational unit, period 2016-2017]. Quito: Universidad Central del Ecuador. http://www.dspace.uce.edu.ec/handle/25000/11523

Cubillo-Arribas, J., Martín-Gutiérrez, S., Castro-Gil, M., \& Colmenar-Santos, A. (2014). Recursos digitales autónomos mediante realidad aumentada [Autonomous digital resources through augmented reality]. RIED. Revista Iberoamericana de Educación a Distancia, 17(2), 241-274. doi:https://doi.org/10.5944/ried.17.2.12686 
Revista Arbitrada Interdisciplinaria KOINONIA

Año 2020. Vol V. N5. Especial II: Educación

Hecho el depósito de Ley: FA2016000010

ISSN: 2542-3088

FUNDACIÓN KOINONIA (F.K). Santa Ana de Coro. Venezuela.

Rodrigo Giovani Aguirre-Herráez; Darwin Gabriel García-Herrera; Claudio Fernando Guevara-Vizcaíno; Juan Carlos Erazo-Álvarez

Fombona-Cadavieco, J., \& Pascual-Sevillano, M. (2016). La producción científica sobre Realidad Aumentada, un análisis de la situación educativa desde la perspectiva SCOPUS [The scientific production on Augmented Reality, an educational literature review in SCOPUS]. EDMETIC, 6(1), 39-61. https://doi.org/10.21071/edmetic.v6i1.5807

Henne, P. I., \& Schmidt-Kraepelin, M. (2015). Benefits of augmented reality in educational settings: a systematic review of the literature. Internationale Tagung Wirtschaftsinformatik, 1542-1556.

Hernández-Revelo, J. (2018) Tutorial para la asignatura control numérico computalizado mediado por simulación computarizada utilizando realidad aumentada. [Tutorial for the subject computerized numerical control mediated by computerized simulation using augmented reality]. Quito UISRAEL, Maestría Telemática Quito: Universidad Israel 2018, 122p. MSc. Mario Oswaldo Basurto Guerrero UISRAEL-EC-MASTERTELEM-378-242-2018-009. https://n9.cl/zpet7

López, A. V. (2014). Aplicación de realidad aumentada orientada a la publicidad de alto impactoen la empresa VECOVA CÍA. LTDA. [Augmented reality application oriented to high impact advertising in the company VECOVA CÍA. LTDA]. Ambato: Universidad Regional Autónoma de los Andes. http://dspace.uniandes.edu.ec/handle/123456789/3335

Marín-Díaz, V., \& Sampedro-Requena, B. E. (2020). La Realidad Aumentada en Educación Primaria desde la visión de los estudiantes. [Augmented Reality in Primary Education from the students' perspective]. Alteridad, 15(1), 61-73. https://doi.org/10.17163/alt.v15n1.2020.05

Melo-Bohórquez, I. M. (2018). Realidad aumentada y aplicaciones [Augmented reality and applications]. Tecnología Investigación Y Academia, 6(1), 28-35.

Méndez-Muros, S. (2018). Meta tecnología, meta-cognición y percepción del uso de las TIC en el aprendizaje universitario. [Meta Tecnologia, goal-cognition and perception of the use of TIC in university learning]. Educación con tecnología un compromiso social, 37-43. https://n9.cl/a22b

Organista-Sandoval, J., Domínguez, P. C., \& López, O. M. (2018). Desarrollo y aplicación de contenidos educativos digitales desde un teléfono inteligente para un tema de Estadística en un curso universitario. [Development and application of digital educational content from a smartphone for a Statistics topic in a university course]. Actuaidades investigativas en educación, 19(1);, 2-23. 
Revista Arbitrada Interdisciplinaria KOINONIA

Año 2020. Vol V. N5. Especial II: Educación

Hecho el depósito de Ley: FA2016000010

ISSN: 2542-3088

FUNDACIÓN KOINONIA (F.K). Santa Ana de Coro. Venezuela.

Rodrigo Giovani Aguirre-Herráez; Darwin Gabriel García-Herrera; Claudio Fernando Guevara-Vizcaíno; Juan Carlos Erazo-Álvarez

Pérez-Lisboa, S., Ríos-Binimelis, C. G., \& Castillo Allaria, J. (2020). Realidad Aumentada y stellarium: astronomía para niños y niñas de cinco años. [Augmented Reality and stellarium: astronomy for five-year-old boys and girls]. Alteridad, 15(1), 25-35. https://doi.org/10.17163/alt.v15n1.2020.02

Prendes-Espinoza, C. (2015). Realidad aumentada y educación: análisis de experiencias prácticas. [Augmented reality and education: analysis of practical experiences]. Pixel-Bit. Revista de Medios y Educación, 46; 187-203.

Quizhpi-Lupercio, L. (2018). "La estrategia de gamificación y el proceso de aprendizaje" [The gamification strategy and the learning process]. Ambato: Universidad Técnica de Ambato. https://n9.cl/wh5ac

Rico-González, G. A., \& Quevedo, T. W. (2016). Aplicación de realidad aumentada para fortalecer las competencias las competencias en el área del dibujo técnico los estudiantes de instituciones de educacion superior. [Application of augmented reality to strengthen the competences in the area]. Ambato: Pontificia Universidad Católica del Ecuador Sede Ambato. https://n9.cl/s5gd

Varela, F., Muñoz, M. C., \& Flores, J. (2018). AR Cocoa: diseño e implementación de siete estaciones de realidad: aumentada en el campus de la Universidad San Francisco de Quito. [AR Cocoa: design and implementation of seven reality stations: augmented on the campus of the San Francisco University of Quito]. INNOVA Research Journal, 3(3), 158-171. https://doi.org/10.33890/innova.v3.n3.2018.492

Zamora, F. R. (2016). Docente su rol y modelo en procesos pedagógicos. [Teacher, his role and model in pedagogical processes]. Ingenio, 1(2); 1-10. 